\title{
Coronavirus (COVID-19) Assessments and the Importance of Calculating the Probability of Illness
}

\author{
Steven D. Stovitz ${ }^{1}$ \\ Published online: 22 April 2020 \\ (C) International Association of Medical Science Educators 2020
}

Since the manuscript "The inability to calculate predictive values: an old problem that has not gone away" [1] was submitted, the coronavirus, COVID-19, pandemic has begun. Many parts of the world are following human quarantine practices to stop the spread of this novel virus. Restaurants are closed, airports are shut down, and medical communities are scrambling to decide who has the virus and who does not. Decisions about who should be quarantined and who should not are implicitly based on the probability of illness. Should we quarantine someone with a 1 in 5 probability for the illness? What about 1 in 10 ? or 20 ? or 50 ? The probability (risk) at which we should set our level for quarantine is unclear and a moving target. Yet, when we make an assessment to quarantine, we are, in essence, judging the person's risk of having COVID-19.

Sadly, tests for the virus are not readily available. Furthermore, when tests are available, they are imperfect. A few studies suggest that a nasopharyngeal swab applied to the reverse transcription polymerase chain reaction test (RT-PCR) has a sensitivity of approximately $70 \%$ [2-4]. This is an unstable estimate based on just a few samples. The sensitivity may differ based on the patient's upper respiratory symptoms (e.g., increased sensitivity with larger amounts of nasal secretions), and the actual sensitivity may also vary as testing procedures evolve. The previous article [1] documented that clinicians and medical students continue to have problems calculating predictive values (i.e., the probability of illness) when given questions from the medical archives. The purpose of this letter is to demonstrate that the ability to make the calculations can affect our decisions during the current pandemic.

Steven D. Stovitz

stovitz@umn.edu

1 Department of Family Medicine and Community Health, University of Minnesota Medical School, 420 Delaware St. SE, MMC 381, Minneapolis, MN 55455, USA
Consider the following situation and the assumptions that among patients who truly have COVID-19, 70\% test positive (70\% sensitivity), and that among patients who truly do not have COVID-19, 100\% test negative (100\% specificity):

You are evaluating a patient, and due to the prevalence in your community and this patient's mild symptoms, you estimate that the person's probability for COVID19 is 1 in 10, i.e. $10 \%$. The person is then tested, and the test result is negative. Does this person need to be quarantined (i.e. beyond your community's standard)?

The probability of illness is shown in Table 1 below using a pretest probability of $10 \%$. As can be seen, given the test characteristics of $70 \%$ sensitivity and $100 \%$ specificity, a person with a 1-in-10 (10\%) pre-test probability and a negative test has a 1-in-31 probability of having COVID-19.

Might this actual calculation matter? Here are two broad scenarios (based loosely on personal conversations with clinicians) where it would matter:

- Clinician A believes that people should be quarantined if their probability is $\geq 1$ in 50 , yet also believes that a negative test means that a person does not have the illness. This clinician would mistakenly not quarantine the individual even though the probability for COVID-19 is greater than 1 in 50.

- Clinician B believes that people need not be quarantined if their probability is $\leq 1$ in 20 . Clinician B also understands that the tests are not perfect; some people with a negative test may have the disease. Given the negative test in someone with a pre-test probability of 1 in 10 , clinician B recognizes that the risk is now $<1$ in 10 , but is unsure how to make the calculation of the post-test probability. Clinician $\mathrm{B}$ wants to be especially cautious and chooses to quarantine the individual even though the probability for COVID-19 is less than 1 in 20. 
Table 1 Contingency table for a test applied to a population of 100 people with a pre-test probability of $10 \%$, a sensitivity of $70 \%$, and a specificity of $100 \%$. In this scenario, a person with a negative test has a 1-in-31 probability of having COVID-19

\begin{tabular}{lccc}
\hline & Has COVID-19 & $\begin{array}{l}\text { Does not have } \\
\text { COVID-19 }\end{array}$ & $\begin{array}{l}\text { Probability of } \\
\text { COVID-19 }\end{array}$ \\
\hline Test positive & 7 & 0 & \\
Test negative & 3 & 90 & $3 / 93(1$ in 31) \\
Total & 10 & 90 & \\
\hline
\end{tabular}

Moving forward in trying to tackle the pandemic, scientists may develop tests with higher sensitivity. Certainly, the prevalence of disease (pre-test probability) is changing by the day, going up in certain areas and down in others. With these changes in prevalence, our tolerance for the risk level at which to quarantine may also change. And, it is perfectly understandable for clinicians to be overly cautious in light of imperfect information. What does not change is the formula for the posttest probability (predictive value), and that applying the formula may affect clinical decisions. I encourage educators to develop novel strategies for teaching clinicians the calculations so that our recommendations will be consistent with our judgments.

\section{Compliance with Ethical Standards}

There was no need for informed consent, and the content is exempt from IRB review.

Conflict of Interest The author declares that he has no conflict of interest.

\section{References}

1. Stovitz SD. The inability to calculate predictive values: an old problem that has not gone away. Med Sci Educ. 2020. https://doi.org/10. 1007/s40670-020-00954-9.

2. Wang W, Xu Y, Gao R, Lu R, Han K, Wu G, Tan W. Detection of SARS-CoV-2 in different types of clinical specimens. JAMA 2020. Available at: http://www.ncbi.nlm.nih.gov/pubmed/32159775. Accessed 21 March 2020.

3. Lippi G, Simundic A-M, Plebani M. Potential preanalytical and analytical vulnerabilities in the laboratory diagnosis of coronavirus disease 2019 (COVID-19). Clin Chem Lab Med 2020. Available at: http://www.ncbi.nlm.nih.gov/pubmed/32172228. Accessed 23 March 2020.

4. Ai T, Yang Z, Hou H, Zhan C, Chen C, Lv W, Tao Q, Sun Z, Xia L. Correlation of chest CT and RT-PCR testing in coronavirus disease 2019 (COVID-19) in China: a report of 1014 cases. Radiology 2020: 200642. Available at: http://pubs.rsna.org/doi/10.1148/radiol. 2020200642. Accessed 22 March 2020.

Publisher's Note Springer Nature remains neutral with regard to jurisdictional claims in published maps and institutional affiliations. 